\title{
SIMULATING THE DIFFUSION OF TECHNOLOGICAL INNOVATION WITH AN INTEGRATED HYBRID AGENT-BASED SYSTEM DYNAMICS MODEL
}

\author{
Chris Swinerd \\ Centre for Simulation and Analytics, \\ Cranfield University, \\ Defence Academy of the UK \\ Shrivenham, Swindon SN6 8LA, UK. \\ c.swinerd@cranfield.ac.uk
}

\author{
Ken R McNaught \\ Centre for Simulation and Analytics, \\ Cranfield University, \\ Defence Academy of the UK \\ Shrivenham, Swindon SN6 8LA, UK. \\ k.r.monaught@cranfield.ac.uk
}

\begin{abstract}
The potential of hybrid models to enhance simulations of the real world is explored. Whilst the scope for design of such models is large, the focus here brings together agent-based and system dynamics modelling within a defined architectural framework. Comprising a number of modules, each of which is implemented in a single modelling paradigm, the design of hybrid models looks to exploit the potential from a range of approaches and tools. Coded within a single programming environment, the international diffusion of technological innovation is used as a case study to highlight hybrid simulation model design and implementation. An integrated hybrid simulation design which incorporates feedback between modules in a continuous, fluid, process is employed to develop a model comprising two system dynamics modules and one agent-based module. The predictions from the hybrid model are compared to known outcomes regarding the national adoption of mobile telephony, fixed internet and fixed broadband. We conclude with some thoughts on the design of hybrid simulation models.
\end{abstract}

Keywords: Simulation, System dynamics, Agent-based, Hybrid modelling, Diffusion

\section{INTRODUCTION}

"If we seek to operate in the real world, it is surely better to have available a range of approaches and tools that draw on the breadth of systems thinking" (Lane, 1994: 118). Whilst the focus of this paper is to introduce the design and implementation of a hybrid simulation model, the longer-term hypothesis being investigated by the authors is that there are circumstances where hybrid modelling enhances simulation outcomes compared to that achieved with a single modelling approach. In particular, the combination of agent-based (AB) and system dynamics (SD) modelling is being explored since most research in this area has concentrated on the discrete event simulation (DES) and SD combination, e.g. Chahal, Eldabi and Young (2013).

Studies, such as that of Wakeland et al (2004) and Rahmandad and Sterman (2008), compare and contrast simulation modelling paradigms. For example, Wakeland et al. (2004) report on their investigation into the use of $\mathrm{SD}$ and $\mathrm{AB}$ modelling for cellular receptor dynamics and their comparative potential for use in education or for planning experiments. While they did not find a simple dividing line indicating when one paradigm or the other would be clearly preferred, they did note that these techniques were complementary, observing that SD is a more natural choice for highly aggregated modelling, while $\mathrm{AB}$ is better suited to studying phenomena at the level of individuals. 
They also concluded that SD models represent the relationships between variables very effectively while agent-based models force more careful consideration of the definition of agents and the specification of their behavioural rules. AB models are better suited to spatial representation and are able to portray heterogeneous / diverse populations more easily.

Rahmandad and Sterman (2008) compare AB and differential equation based modelling methods using contagious disease as a case study. Their experimental design explores the impact of social network structure and individual heterogeneity on comparative performance of these modelling approaches. They show that social clustering and individual heterogeneity can cause differences in simulation outcomes. However, they reflect that the impact of such differences depends on the purpose of the model.

Whilst it is necessary to understand when one modelling paradigm might be used in preference to another, there may be situations where a hybrid approach that combines paradigms might be more appropriate. In order to implement hybrid modelling solutions, however, design methodologies and tools are required. While tools which support hybrid simulation are becoming more widespread (e.g. Anylogic), the literature contains very little guidance on hybrid model design. The authors aim to address that gap both by examining the nature of hybrid simulations which have appeared in the literature in order to identify design classes for hybrid AB-SD modelling (Swinerd and McNaught, 2012a) and by presenting specific examples or case studies where hybrid simulation may be beneficial, which, along with a discussion of hybrid simulation design, is the rationale for this paper. The domain of international diffusion of high-tech infrastructure was chosen due to its socioeconomic importance and the nature of such diffusion processes which contain both aggregate and discrete elements.

Considering design classes for hybrid simulation models, and drawing from Shanthikumar and Sargent (1983), the authors have outlined three basic types of hybrid AB-SD simulations referred to as sequential, interfaced and integrated classes (Swinerd and McNaught 2012a). Whilst our focus is AB-SD hybrid models, we speculate that the classification presented may also be useful for other classes of hybrid simulations.

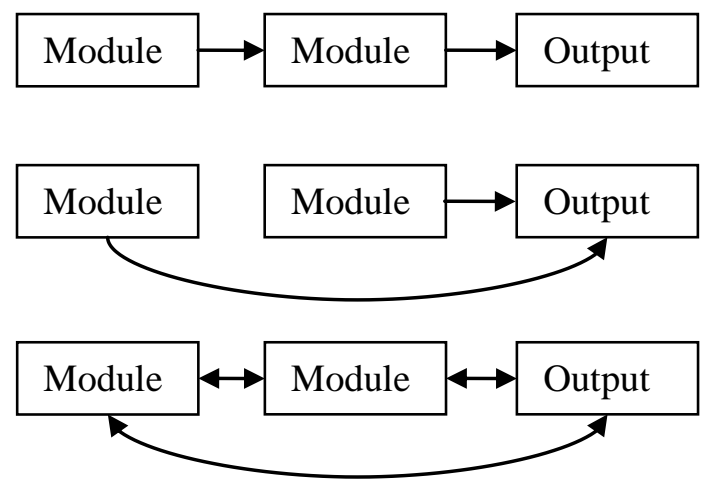


Figure 1 Schematic representation of the Sequential (top), Interfaced (middle) and Integrated (bottom) design classes for hybrid models

With reference to Figure 1, the top schematic represents the architectural construct for a sequential design class. The output of a module is fed to the input of another module, both of which are implemented in a different modelling paradigm, before the final model output is produced by the second module. In the interfaced design class, middle schematic diagram, the output of each module is combined to provide the final model output. In this case modules can be run sequentially or concurrently but they do not directly influence each other. Finally, in the integrated design class, modules and model output are fully integrated with connections between any of them providing the opportunity for feedback and a continuous flow of information.

In contrast to the design classes proposed by Swinerd and McNaught (2012a), Chahal, Eldabi and Young (2013) propose two forms of interactions for hybrid SD-DES models. They introduce these based on the way the two modelling paradigms interact within a model, classifying them as cyclic or parallel. In regards to cyclic interactions, they state (p55): "There are no interactions between SD and DES during run time. They interact with each other only after completion of their individual run." This definition aligns with both the sequential and interfaced design classes and makes no distinction between them. In regards to parallel interactions, they state (p55): "In this mode, SD and DES models are run in parallel while information is exchanged during run time", which aligns to the integrated design class. The two categories of interaction defined by Chahal, Eldabi and Young form part of a conceptual framework for hybrid SD-DES model design in which they assess: 1) the need for a hybrid model; 2) what is to be exchanged between the modelling paradigms; and 3) the nature of interactions as just outlined. Noting the comparison between that reported by Swinerd and McNaught (2012a) and Chahal, Eldabi and Young (2013) and as highlighted by Viana et al (2012), the specific focus of design classes for SD-AB hybrid models and interactions for SD-DES hybrid models could be relaxed to cover a wider combination of modelling paradigms.

Whilst the longer term focus for this research is to determine the circumstances where hybrid modelling enhances simulation outcomes compared to that achieved with a single modelling approach, the aim of the work reported here is specifically to introduce design and implementation of a hybrid AB-SD model. Using national adoption of technological innovation as a case study, a model developed according to the integrated design class is introduced. The model is tested against diffusion data for the international adoption of mobile telephone, fixed internet and fixed broadband technologies. An initial assessment of taking this approach compared to using a single modelling paradigm is provided although this is the subject of ongoing research by the authors.

Before introducing the design and implementation of the model, the concept of coupling different scales of the system is first discussed. Relative to a defined baseline, simulation results are 
then presented and findings assessed against previously published works that consider national traits and adoption behaviours (Craig et al, 1992, Dekimpe et al 1998, Rogers, 2003 and Venkatesh, 2003).

\section{COUPLING SCALES OF THE SYSTEM}

The effective and efficient representation of different scales of a system and the coupling between them is perhaps one of the biggest challenges to single paradigm models. Capturing coherently the detail of individual and aggregate measures within a system as a function of time, space or event can be challenging. Some examples that highlight this challenge are presented here.

In their review of agent based and equation based modelling, Parunak, et al (1998) define unifying multiplicities between individuals and observables of a system whereby individuals can be described by their behaviours and observables by equations.

More recently and specifically in regards to simulation of innovation diffusion, Kiesling et al (2012) review the benefits and potential of agent-based modelling in contrast to more traditional aggregate approaches. They précis a number of limitations reported in the literature associated with the aggregate equation based approach covering: predictive power (i.e. used predominantly for historical analysis), explanatory power (i.e. not behaviourally based), population heterogeneity (i.e. assumed homogeneity with aggregate models), social pressures (i.e. does not breakdown societal effects such as social learning, social pressures or network effects) and prescriptive guidance (i.e. limited potential to explore the effect of decision variables available to managers). They go on to state that many of these limitations can be overcome through "an individual-based modelling approach", i.e. an agent-based approach.

Duggan (2011) introduces an equation based modelling tool which is based on an aggregateindividual-network classification for complex systems. Using an equation based design methodology to combine modelling approaches, Duggan proposes that in using this tool, modellers and stakeholders can transition from aggregate to disaggregate models, gaining insights into the benefits and drawbacks of these approaches for a given problem.

The reviews of Parunak et al (1998), Kiesling et al (2012) and Duggan (2011) all highlight an opportunity to explore "coupling between scales of the system", between aggregate (equation based, homogeneous) and individual (behavioural, heterogeneous) representation of systems. Within a considered architectural design, such as highlighted by Swinerd and McNaught (2012a), hybrid models may provide a solution, bringing together both aspects where required to enhance the overall potential of simulation.

Lorenz and Jost (2006) propose an orientation-framework for multi-paradigm modelling with the aim of aligning purpose, object and methodology. As part of this work, they explore the concept of 'alternative environments' in agent-based modelling: a 'zero' environment with which agents do not interact but which may contain some aggregate parameters for use in an agent model; a 'passive' environment that does not contain any inherent dynamics but with which agents can interact with 
variables or structures; and finally an 'active' environment which is dynamic and an active part of the agent model. They propose that an SD model could be used to build the 'active' environment.

Combining the unified multiplicities of Parunak, et al., with the alternative environments described by Lorenz and Jost, provides a general framework that could be used to capture the decomposition of a model, as illustrated in Figure 2, and clarify the most suitable design class if a hybrid modelling approach is appropriate. Inclusion of the environment in this way also provides the opportunity to represent the context of the study and therefore make apparent some, if not all, objectives of the modelling study, i.e. the environment and interactions with the environment that are of interest. The arrowheads confirm the direction of interactions, where the nature of those interactions is also defined. Ongoing analysis suggests that if the 'individuals' element is included in the design of a model, then there must always be a 'reports' interaction with the 'environment' element. Otherwise, elements and interactions can be incorporated as required although only ever within that presented in Figure 2, i.e. arrowheads should not be added to interactions beyond that defined, but they can be removed as required. Whilst also subject to ongoing analysis, it is anticipated that the design of a model can incorporate multiple instances of 'individuals' and 'observables' elements, providing linked interactions between them are consistent with that defined.

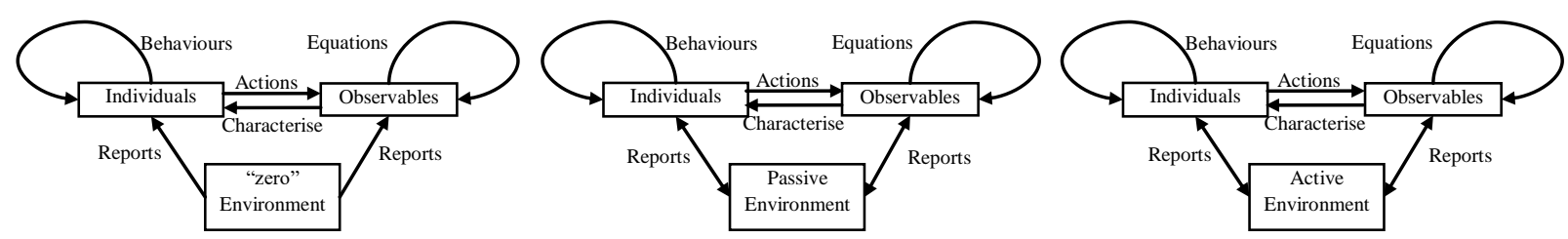

Figure 2 General framework for hybrid modelling

Considering the national adoption of technological innovation, nations are modelled here as individual agents and their behaviour is represented by their adoption status. Diffusion of innovation is observable with equations used to calculate the timing of the process. This hybrid design then links the behaviour of nations to the aggregate diffusion process through the dynamics of the social system, in this case, the international community of Nations; which is an 'active environment'. In line with previous comments on the use of the general framework, the social factors interaction is designed only to 'report' from the 'active environment' element to the 'observables' element; although potentially allowable, the design does not include a direct 'report' in the opposite direction. Whilst the design of this model may be possible in a single paradigm, a hybrid approach exploits the 'natural' benefits of both SD and AB paradigms. Against the proposed general framework for hybrid models, this decomposition is presented in Figure 3. 


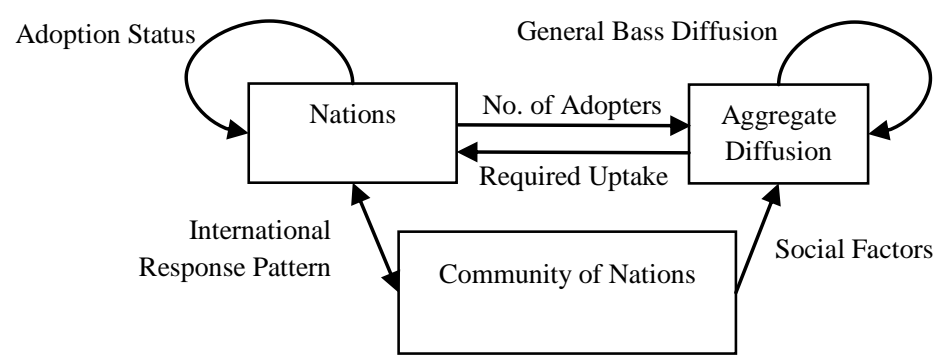

Figure 3 Proposed decomposition for the hybrid model of international technological diffusion

Whilst further research is required, it is proposed that the adoption of the general framework may also support a number of options for conducting verification and validation such as those identified by Sargent (2013). In particular, support for the use of structured programming, programme modularity, tests for proper implementation, data relationship correctness and the reprogramming of critical components of the programme would all be facilitated through the use of the general framework approach. Breaking the model down into key modules and their interfaces as defined in the general framework also supports the potential for code re-use. As Sargent comments (p 12): "several versions of a model are usually developed prior to obtaining a satisfactory valid model", in which case code reuse aids efficiencies in both project time and cost.

\section{DESIGN OF THE MODEL}

The aggregate timing of the diffusion process lends itself to established equation-based rate models, such as those found in the seminal work of Bass (1969). Behaviour arising from individual decision making within a social system, on the other hand, readily lends itself to modelling within an $\mathrm{AB}$ paradigm. This identifies two modules within the model; each offering known advantages with their respective modelling paradigm, i.e. representation of temporal and spatial patterns, respectively.

The final aspect considered in designing this model is how to represent decision making within agents. The established theories of reasoned action (Fishbein and Ajzen, 1975) and of planned behaviour (Taylor and Todd, 1995) provide "the theoretical foundation supporting 'intention to use' as a predictor of usage" in technology acceptance models (Hamre, 2008). These equation-based theories define weighted combinations of internal and external influences which represent the fluidity of decision making and hence suggest the inclusion of another SD module within the model.

Using the style introduced in Figure 1, the outline design framework for the model is first defined and, along with its implementation using three modules mapped against various scales of the system, is presented in Figure 4. 

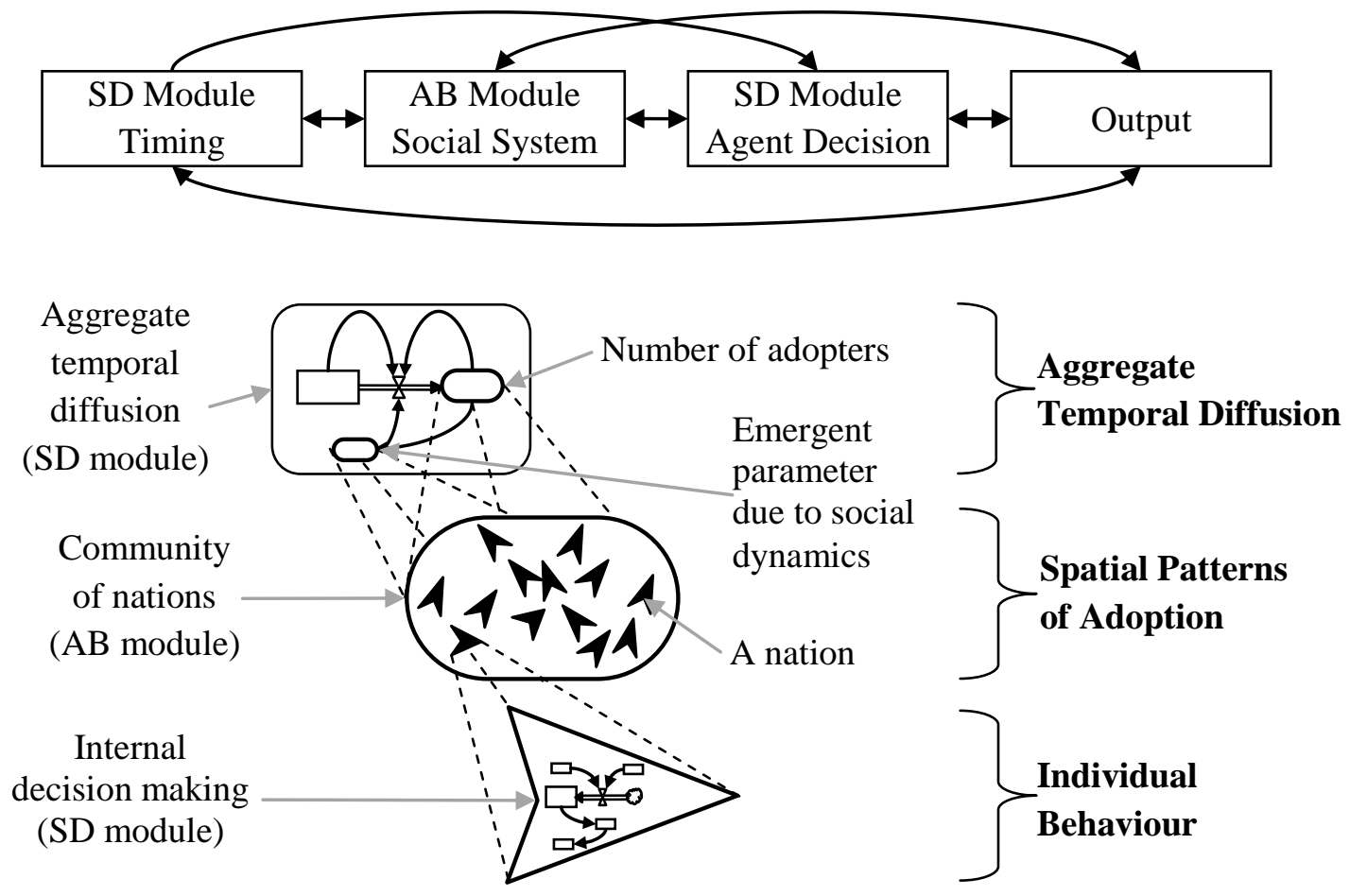

Figure 4 Architectural implementation of the model using three modules; two SD and one $A B$

With reference to Figure 4, the SD module of aggregate diffusion controls the timing of the process, defining the number of anticipated adopters at each time step. The pattern of diffusion amongst the community of nations is allowed to evolve until the number of nations that have adopted reaches the aggregate expectation. Individual nations decide whether to adopt on the basis of their own intentions and the behavioural influence of other nations. Aggregate measures of the social response can then be fed back to the timing module. For now, the adoption fraction is used but inclusion of the $\mathrm{AB}$ module allows for other measures to be explored with respect to informing the SD timing module. This design, therefore, couples aggregate prediction and individual behaviour in a coherent fashion within the context of a social system, a construct that is clearly captured in the general framework shown in Figure 3.

\section{IMPLEMENTING THE MODEL}

Drawing from Sterman's system dynamics interpretation (Sterman, 2000: 332-334) of the Bass diffusion model, Bass (1969), the aggregate adoption rate, $\mathrm{R}_{\mathrm{A}}$, comprises the sum of adoptions arising from external influences, such as advertising, and those arising from social dynamics, such as wordof-mouth. The contribution of these influences are controlled by coefficients of innovation and contact rate, $\alpha$ and $\mathrm{c}$, and the adoption fraction, $\beta$, which is relative to the size of the sample population $m$. As in Figure 5, global variables as shown within angular ' $<>$ ' parenthesis. 


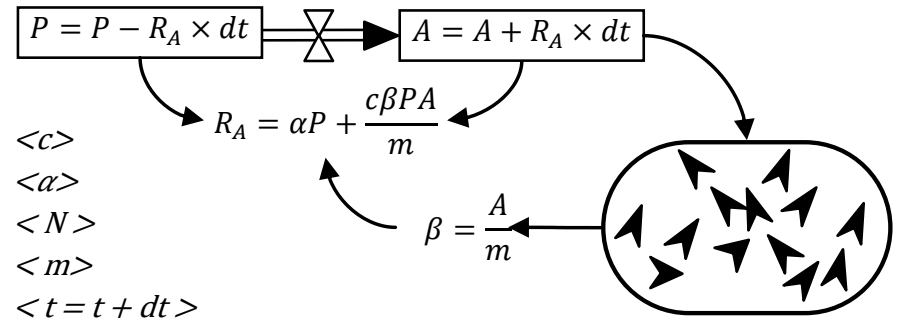

Figure 5 The aggregate timing module and interface to the $A B$ module

The adoption rate determines the numbers of adopters, $A$, and potential adopters, $P$, in the sample population as a function of time. The unit of time for analysis is years as available data against which to validate the model is published annually. The time step for simulations was fixed at $d t=$ 0.25 for the aggregate diffusion module. Time is managed only at this level in the model, advancing only when sufficient nations in the agent-based module have adopted for that time step.

Within each nation, a system dynamics module represents the decision making process as shown at Figure 6. The pressure to adopt, $P r$, is determined at each simulation step, $s$, by the rate of change in pressure, $R_{p}$. Drawing from the theory of reasoned behaviour, pressure comprises the weighted sum of internal pressure and the subjective norm, external bandwagon pressure, related to that internal pressure.

Internal pressure to adopt, $I P$, is represented using national development indicators drawn from the World Bank's databank (World Bank, 2012). A static average of that reported over the period 1980 to 2009 , which aligns to the adoption of the technologies considered, is used for each indicator in order to maximise the number of nations represented. The sample population for a simulation is, therefore, made up of only those nations that have a recorded value for both national development indicators being considered.

Accounting for bias, such as might arise from measures influenced by population or land mass, for example, 42 indicators, $M_{i}$ (i.e. $M_{1}$ to $M_{42}$ ), were selected which represented the 26 generalisations defined by Rogers (2003) for adopter categories and innovativeness. Covering social, economic, and environmental measures, these indicators capture the individuality of agents (nations) within the social system.

In order to readily combine internal and external pressure, national indicators were normalised relative to the maximum value of that indicator amongst the sample population for the simulation. Internal pressure is then represented through a weighted pairwise contribution of national indicators.

Because some national measures have a wide dynamic range, covering up to six orders of magnitude, the internal iteration of pressure, $d s$, is adjusted dynamically in order to reduce computation time. Controlled with a scaling factor, $a$, this adjustment is relative to the number of simulation interactions, $S$, between time steps, $d t$, and a practical limit imposed on simulation 
iterations, $L$. At the start of each time step controlled by the SD timing module, $d s=0.1$ for all nations. Therefore, the overall progression of time is event driven, advancing only when sufficient nations have adopted to meet the aggregate requirement. Of course, the detailed patterns of adoption are only captured in the agent module, reflecting both the individual intention of nations and the influence of international bandwagon pressure.

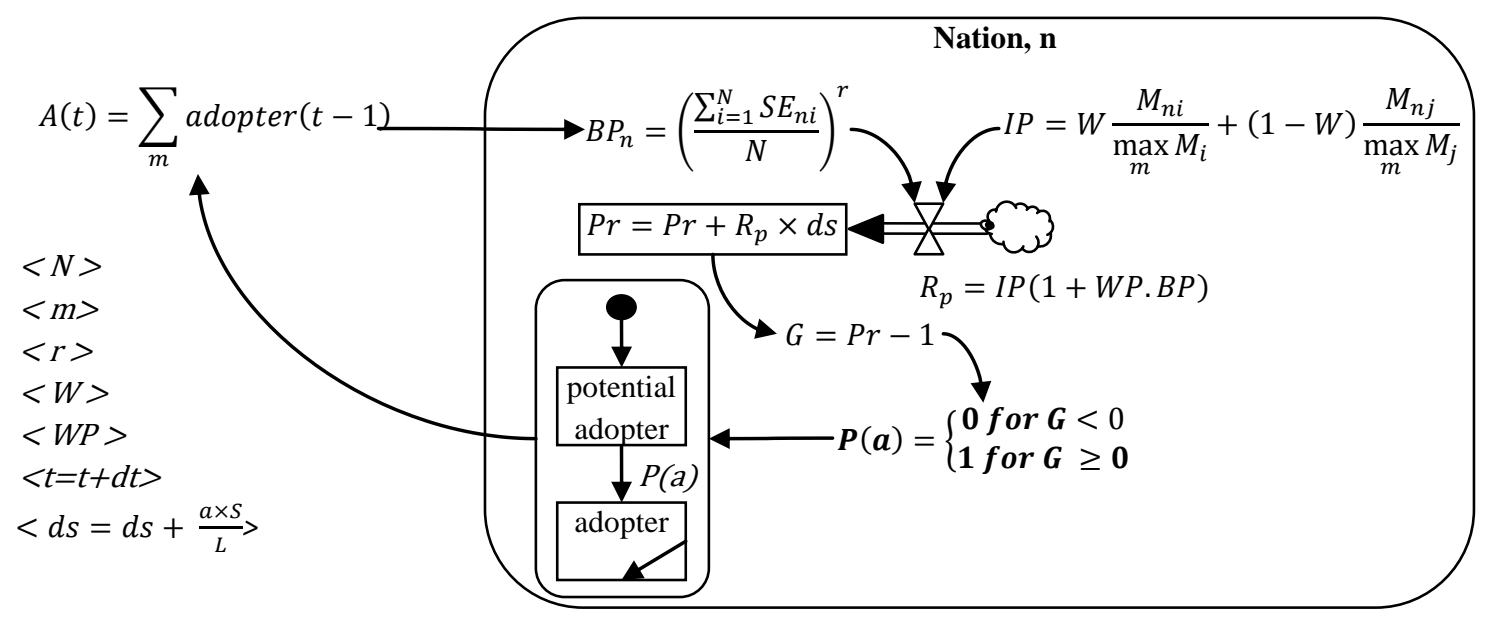

Figure 6 The decision making SD module and interface with other agents in the AB module

Social influence in the form of external bandwagon pressure, $B P$, is represented here as a weighted proportion of neighbours who have adopted. On the importance of social influence as a function of system age, Venkatesh et al (2003: 453) state: "This normative pressure will attenuate over time as increasing experience provides a more instrumental (rather than social) basis for individual intention to use the system." Bandwagon pressure may, therefore, be more important earlier in the diffusion process and so a shaping parameter, $r$, is applied. The strength of influence between nations is represented through structural equivalence theory, calculated in accordance with Sailer (1978). Here, structural equivalence, $S E$, was determined on the basis of shared language, using only indigenous languages spoken by at least $10 \%$ of the national population. In order to account for the findings of Craig et al (1992), the geographic extent of social influence can be constrained such that $N$ is the number of neighbours who can influence each nation, $n$, selected on the basis of those nearest. The position of each nation is represented as an estimate of its geographic centre based on defined borders for the political map of the World represented in Figure 10. Nearest neighbours are those with smallest radial separation. The authors are exploring alternative representations of social influence including the influence from nations yet to adopt and alternative measures for structural equivalence such as using measures of bilateral trade, for example.

With the large dynamic range of some national indicators, the combination of internal and external pressure is balanced. A weighting factor, $W P$, can then be used to investigate the relative contributions of each. 
As pressure is perceived, the decision for a nation to adopt was defined relative to a gap between the building pressure, $P_{r}$, and a decision threshold, $T_{n}$, where $T_{n}=1$ for all nations. This gap informs the probability of adopting, $P(a)$, at each iteration, s, of the simulation with two forms of decision making investigated; binary and the logit function often used to represent discrete choice.

The model is implemented wholly within Netlogo (4.0.3), (Wilensky, 1999), and, therefore, interfacing different modelling tools is not an issue. Coding within a single programming environment enables the researcher to focus on the integral design of the model. As highlighted by Swinerd and McNaught (2012a) and also through the earlier example of Duggan's work, there are a range of tools that can be considered for implementing hybrid AB-SD models.

\section{DEFINING A BASELINE}

In order to baseline the effectiveness of the model, the non-parametric measure of Spearman's rank order correlation, $r_{s}$, between internal pressure, $I P$, only and the year of national adoption for each of the three technologies was calculated; using different values of $W$ to weight the relative contribution of either national indicator as defined in Figure 6. The national year of adoption was defined as the first year in which subscribers are reported in the WorldBank's dataBank. The baseline results are given in Table 1. In order to contrast the pairwise baseline results, those achieved for single national indicators are also listed. Of the 209 nations for which data is held, the number of nations, i.e. the sample population $m$, represented is 53,96 or 114 . Sample population size is determined by the availability of both national indicators and knowing the actual year of adoption.

Table 1 Baseline rank order correlation for combinations of national indicators

\begin{tabular}{|l|c|c|c|l|l|}
\hline \multicolumn{6}{|l|}{ Single National Indicator Assessment } \\
\hline Technology & $r_{s}$ & $m$ & $M$ \\
\hline Mobile Telephones & 0.710 & 128 & Daily newspapers (per 1,000 people) \\
\hline Fixed Internet & 0.754 & 187 & \multicolumn{2}{l|}{ Scientific and technical journal articles } \\
\hline Fixed Broadband & 0.680 & 114 & \multicolumn{2}{l|}{ Average years of schooling of adults (aged 15+) } \\
\hline Weighted Pair Wise Combination Assessment & $M_{j}$ \\
\hline Technology & $r_{s}$ & $m$ & $W$ & $M_{i}$ & $\begin{array}{l}\text { Roads, passengers carried } \\
\text { (million passenger-km) }\end{array}$ \\
\hline Mobile Telephones & 0.862 & 53 & 0.2 & $\begin{array}{l}\text { Daily newspapers (per } \\
1,000 \text { people) }\end{array}$ & $\begin{array}{l}\text { Researchers in R\&D (per } \\
\text { million people) }\end{array}$ \\
\hline Fixed Internet & 0.845 & 96 & 0.9 & $\begin{array}{l}\text { Total reserves (includes } \\
\text { gold, current US\$) }\end{array}$ & $\begin{array}{l}\text { Total reserves (includes } \\
\text { gold, current US\$) }\end{array}$ \\
\hline Fixed Broadband & 0.801 & 114 & 0.1 & $\begin{array}{l}\text { Average years of schooling } \\
\text { of adults (aged 15+) }\end{array}$ \\
\hline
\end{tabular}


The work of Dekimpe, et al (2000) on global adoption timing of technology across countries provides a useful cross-reference here. They conclude that population concentration is the most important predictor of a country's adoption timing and define the profile of an innovative country as: "one that is wealthy (on a per capita basis), has a highly concentrated population, is open (part of the global economy) and is culturally homogeneous." It is also worth noting that they recognise the influence of nations who have adopted on potential adopter nations who are 'similar'; which is relevant to structural equivalence. More generally, Rogers considers socioeconomic, personality and communication traits (Rogers, 2003: 287 - 293) that define innovativeness. More relevant at the national level, important socioeconomic and communications indicators are: years of formal education; literacy; social status (wealth, employment, social divide, etc.); social mobility; larger sized units (farms, companies, schools etc.); social participation; connectivity of interpersonal networks; being cosmopolitan; exposure to mass media; exposure to interpersonal communication channels; information seeking; increased knowledge and opinion leadership. The baseline results, therefore, share much in common with previous findings, although lack the explicit contribution of social dynamics for which it was anticipated that modelling would enhance prediction beyond baseline.

\section{SIMULATION RESULTS}

The aggregate pattern of diffusion over time was first assessed. As presented by Dekimpe et al (1998: 116 - 117), a significant factor to consider is "truncation bias" which can occur when comparing diffusion curves. In order to contrast features within the diffusion process without temporal bias, comparisons should be made as a function of system age, i.e. time elapsed since the diffusion process started. As a function of system age, i.e. all adopters timed from the year that subscribers are first recorded, the coefficients for aggregate diffusion for each of the three technologies were calculated using the method of least squares error to fit simulation to reference data as shown in Figure 7. For mobile telephone, fixed internet and fixed broadband the coefficients of innovation, $\alpha$, and contact rate, $c$, were $0.04,0.09$ and 0.12 and $0.45,0.9$ and 0.7 respectively. 


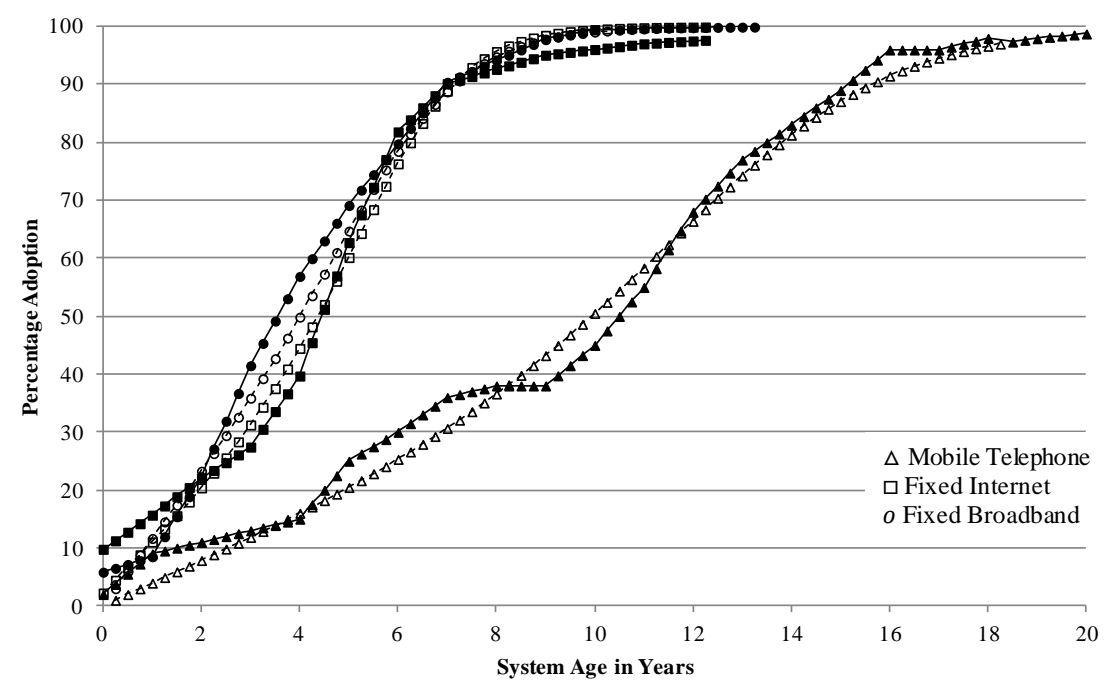

Figure 7 Aggregate diffusion curves showing the simulated fit (dashed) to the actual data (solid)

It is likely that the reference data for fixed internet is not entirely correct as the adoption process starts at ten percent.

The hybrid model was then run, varying the control parameters of the model $(W, W P, r$ and percentage geographic constraint, $\% G$ ) for a full pairwise assessment of national indicators. The model was also configured to compare results for decision making with the binary or the logit formulation and also with and without structural equivalence. The utility of a heuristic search methodology using an evolutionary process to determine the values for model control parameters has been reported by Swinerd and McNaught (2012b).

For all three technologies it was possible to obtain Spearman rank order correlations higher than the weighted pairwise baseline results. Whilst these individual increases are not statistically significant, which is not surprising given the high baseline values, there is consistent improvement for each of the three technologies modelled here. Table 2 summarises the top performing configurations for the model. There were three configurations which equally produced the best outcome for mobile telephones without structural equivalence included.

Table 2 Model configurations providing best simulation outcome

\begin{tabular}{|c|c|c|c|c|c|c|c|c|c|c|c|c|c|}
\hline Technology & $r_{s}$ & $m$ & t-test & $\alpha$ & $c$ & $M_{i}$ & $M_{j}$ & $\begin{array}{l}\text { Decision } \\
\text { Making }\end{array}$ & $S E$ & $W$ & $W P$ & $r$ & $\% G$ \\
\hline \multirow{4}{*}{ Mobile Telephone } & 0.878 & \multirow{4}{*}{53} & 0.449 & \multirow{4}{*}{0.04} & \multirow{4}{*}{0.45} & \multirow{4}{*}{$\begin{array}{l}\text { Daily news papers } \\
\text { (per } 1,000 \text { people) }\end{array}$} & \multirow{4}{*}{$\begin{array}{c}\text { Roads, passengers carried } \\
\text { (million passenger-km) }\end{array}$} & \multirow{4}{*}{ Binary } & Yes & 0.2 & 1.2 & 0.3 & 20 \\
\hline & \multirow{3}{*}{0.877} & & \multirow{3}{*}{0.420} & & & & & & \multirow{3}{*}{ No } & 0.2 & 0.6 & 0.3 & 10 \\
\hline & & & & & & & & & & 0.2 & 1 & 0.3 & 10 \\
\hline & & & & & & & & & & 0.2 & 1.4 & 0.3 & 10 \\
\hline \multirow{2}{*}{ Fixed Internet } & 0.884 & \multirow{2}{*}{53} & 1.060 & \multirow{2}{*}{0.09} & \multirow{2}{*}{0.9} & \multirow{2}{*}{$\begin{array}{l}\text { Daily news papers } \\
\text { (per } 1,000 \text { people) }\end{array}$} & \multirow{2}{*}{$\begin{array}{c}\text { Roads, passengers carried } \\
\text { (million passenger-km) }\end{array}$} & \multirow{2}{*}{ Binary } & Yes & 0.2 & 1.8 & 0.1 & 10 \\
\hline & 0.868 & & 0.590 & & & & & & No & 0.2 & 1.8 & 0.1 & 10 \\
\hline \multirow[b]{2}{*}{ Fixed Broadband } & 0.802 & \multirow[b]{2}{*}{114} & 0.019 & \multirow[b]{2}{*}{0.12} & \multirow[b]{2}{*}{0.7} & \multirow{2}{*}{$\begin{array}{l}\text { Average years of } \\
\text { schooling of adults } \\
\text { (aged } 15+)\end{array}$} & \multirow{2}{*}{$\begin{array}{l}\text { Total reserves (includes } \\
\text { gold, current US\$) }\end{array}$} & \multirow{2}{*}{ Binary } & Yes & 0.1 & 0 & 0.1 & 20 \\
\hline & 0.803 & & 0.038 & & & & & & No & 0.1 & 1.6 & 0 & 40 \\
\hline
\end{tabular}


The best model configuration for the fixed internet case was the only one where the national indicators were different to the baseline tests. Interestingly, this used the same combination of national indicators as required for mobile telephones.

The sensitivity of model performance was assessed for each of the parameters in grey cells of Table 2. Each parameter was varied in turn, whilst the other parameters were held to their default value in the greyed cells. The results of this analysis are presented Figure 8.
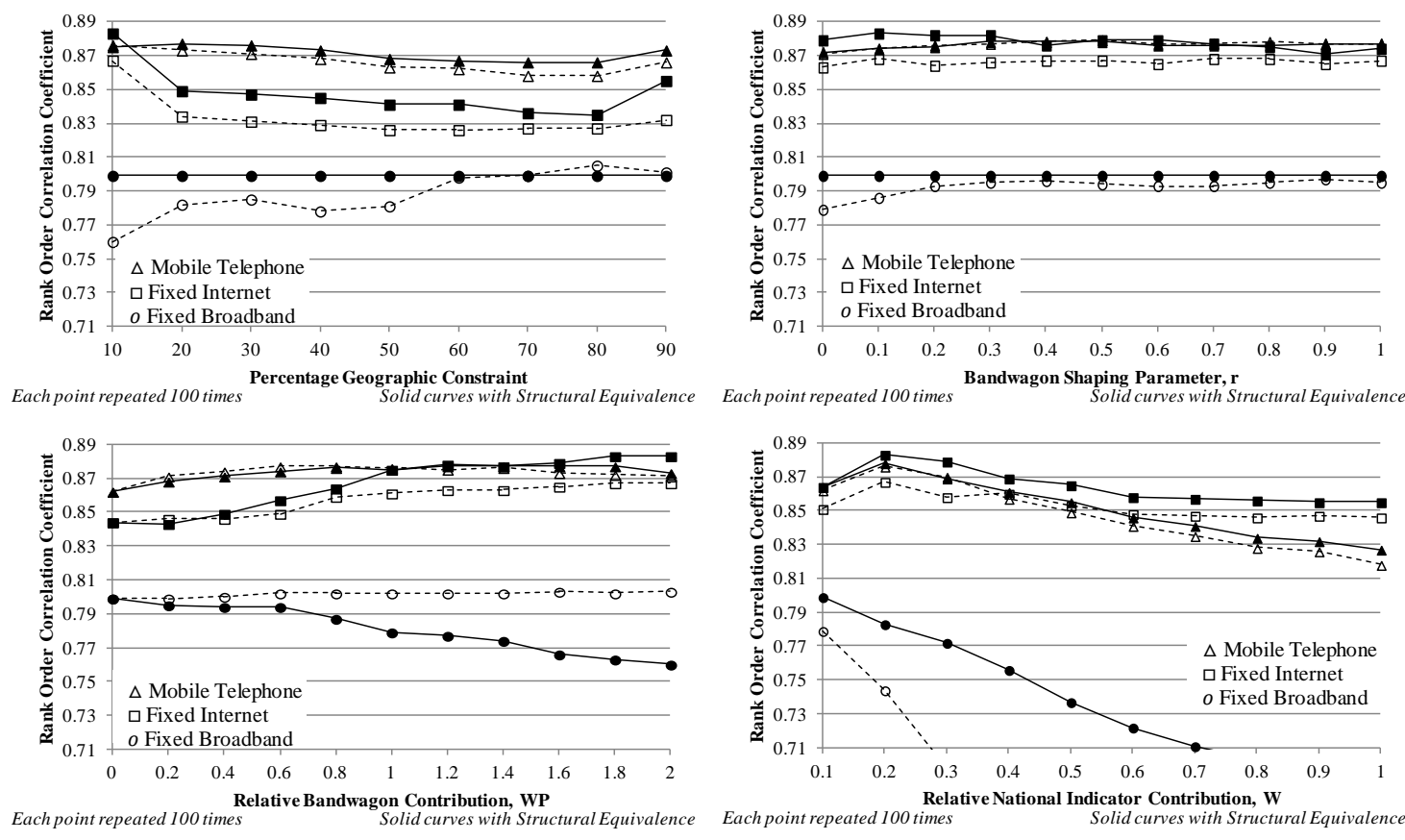

Figure 8 Sensitivity of aggregate simulation outcome to key model parameters and configuration

These results suggest that the diffusion process for fixed broadband is different to the other technologies considered. The results for mobile telephony and fixed internet indicate that weighting internal and external bandwagon pressure, $W P$, is required, suggesting that social dynamics are influential. The same conclusion cannot, however, be drawn for fixed broadband. This may be because this technology is essentially an upgrade, whereas the other two technologies are, in a sense, original innovations.

Geographic constraint can have an influence when constrained to less than $\sim 40 \%$ of the sample population, which aligns with the findings of Craig et al (1992). They concluded from their longitudinal analysis of industrialised nations over the period 1960 to 1988, that: "in spite of the increased communications, travel and trade between countries, physical distance remains an important determinant of macro-environmental similarity."

The shaping parameter, $r$, can have an effect, although not strong. Peak results are obtained for $r<0.3$ indicating that bandwagon pressure may be more important earlier in the diffusion process, which agrees with the observations reported earlier of Venkatesh et al (2003). Again, the upgrade 
analogy for fixed broadband may apply with nations having developed experience through use of the internet and, therefore, not be so influenced by social factors.

Simulation results are sensitive to the weighting applied for combining national indicators, $W$. For mobile telephony and fixed internet the bias is towards the passengers carried by road and for fixed broadband total reserves. The inclusion of structural equivalence typically improves simulations.

Focussing on national representation within the model, Figure 9 shows prediction error in years as a function of actual adoption registered to system age. These results for national adoption of mobile telephony were achieved using the default model configurations with structural equivalence included as defined in Table 2. The fifty three nations represented are identified using the international three digit code designation ISO 3166-1 alpha-3. The scales for prediction error are set for clarity and, therefore, nations falling outside of these ranges are identified bottom left for each plot.

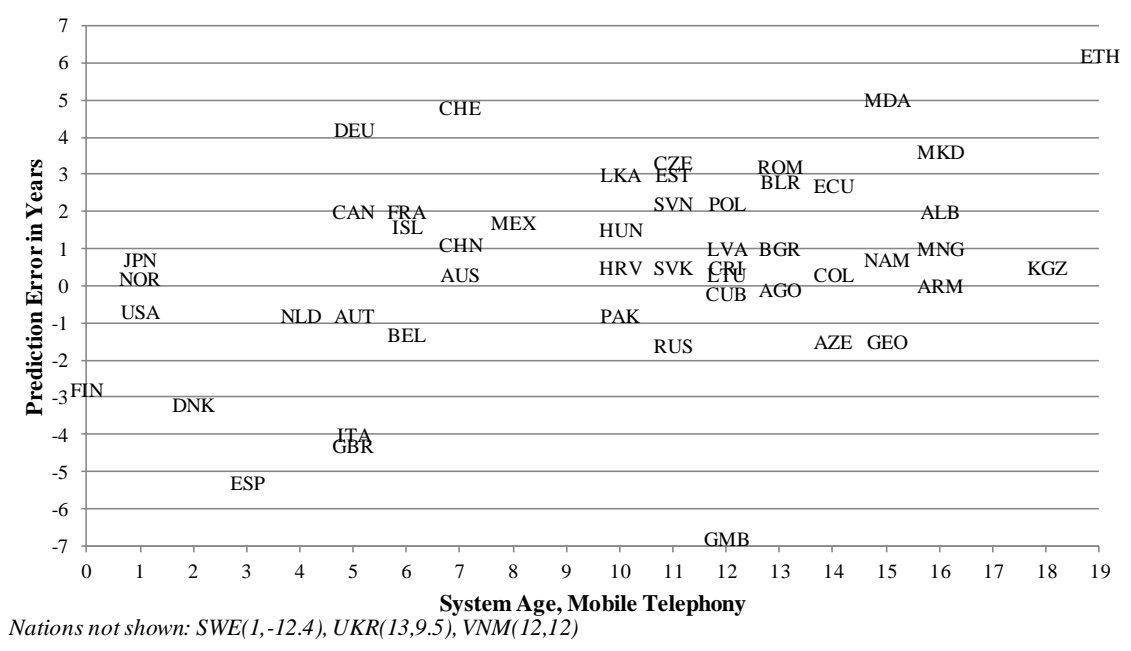

Figure 9 National Representation for the adoption of mobile telephony

An alternative representation of national behaviour is to show the spatial pattern of adoption such as presented in Figure 10 for the adoption of mobile telephony at four snapshots in time. 


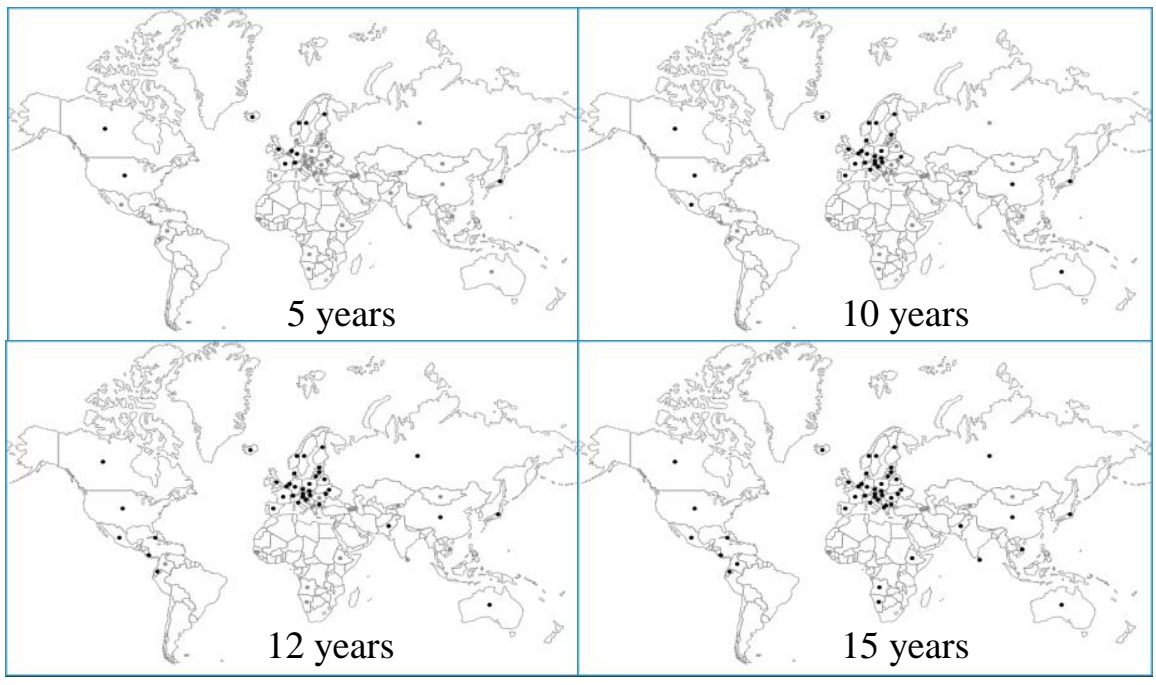

Figure 10 Simulated national adoption for mobile telephony at four snapshots of system age

The results in Figures 9 and 10 show the representation of the nations for which national indicator data was available. They can be assessed coherently with the aggregate results in Figure 7 and, therefore, the coupling between these scales of the system can be investigated easily.

\section{CONCLUSIONS}

In this paper, we have developed a model to simulate the international diffusion of high-tech products. This is an important and interesting domain containing sufficient publicly available data to provide the necessary simulation inputs and to permit comparisons with simulation outputs. The model itself is a hybrid simulation, combining elements of system dynamics and agent-based simulation in a modular structure with feedback present between the different modules.

Results produced by the model to date, making use of available national data, are most encouraging. In addition, more general insights gained from the model show good general agreement with the diffusion literature, e.g. the extent of geographic influence and the tendency for bandwagon pressure to be more important early in the process.

The implementation of this hybrid AB-SD model has clearly demonstrated that model output which depends on a coherent combination of behaviours across different scales of a system, in this case between the aggregate diffusion process and the bandwagon effect on individual national behaviours, can be readily produced. The chosen hybrid model design utilises the natural benefits of two different simulation paradigms. Potentially, however, this model could have been implemented within a single paradigm or by using the agent-oriented SD approach as proposed by Duggan (2011). It remains an interesting question whether the effort required in 'stretching' a single paradigm is worthwhile compared to exploiting the 'natural' benefits of individual modelling paradigms combined in a hybrid 
approach. The answer must depend, in part, on the experience, flexibility and willingness of the modeller to engage with more than one simulation paradigm.

As illustrated in Figure 11, decision making during the early design phase of a model has been facilitated both by capturing the general framework for representing modelling content and mapping this to one of the architectural design classes for hybrid SD-AB models previously proposed by the authors (Swinerd and McNaught, 2012a). It is with respect to these early decisions of the design phase that the authors will focus further work, along with an investigation of other potential benefits of a hybrid simulation design, such as to model verification and validation, as suggested by Sargent (2013).

Although further research is required to assess the benefits of such an approach compared to modelling in a single paradigm, the growing literature concerning hybrid simulation suggests that hybrid designs will find increasing use across a growing number of application domains. However, despite increasing interest in the area, there remains a lack of guidance for potential hybrid simulation modellers concerning how to go about such a task. In this paper, our aim was to demonstrate a novel and useful approach to the general design and implementation of a hybrid SD-AB model. In fact, we believe that this approach is also readily adapted to other hybrid configurations including the discreteevent simulation paradigm. 

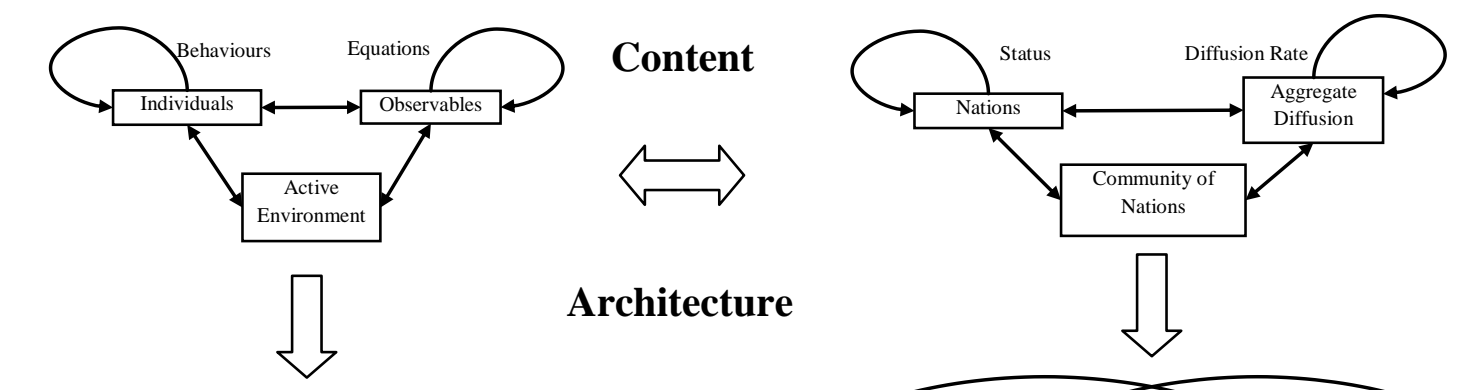

Architecture
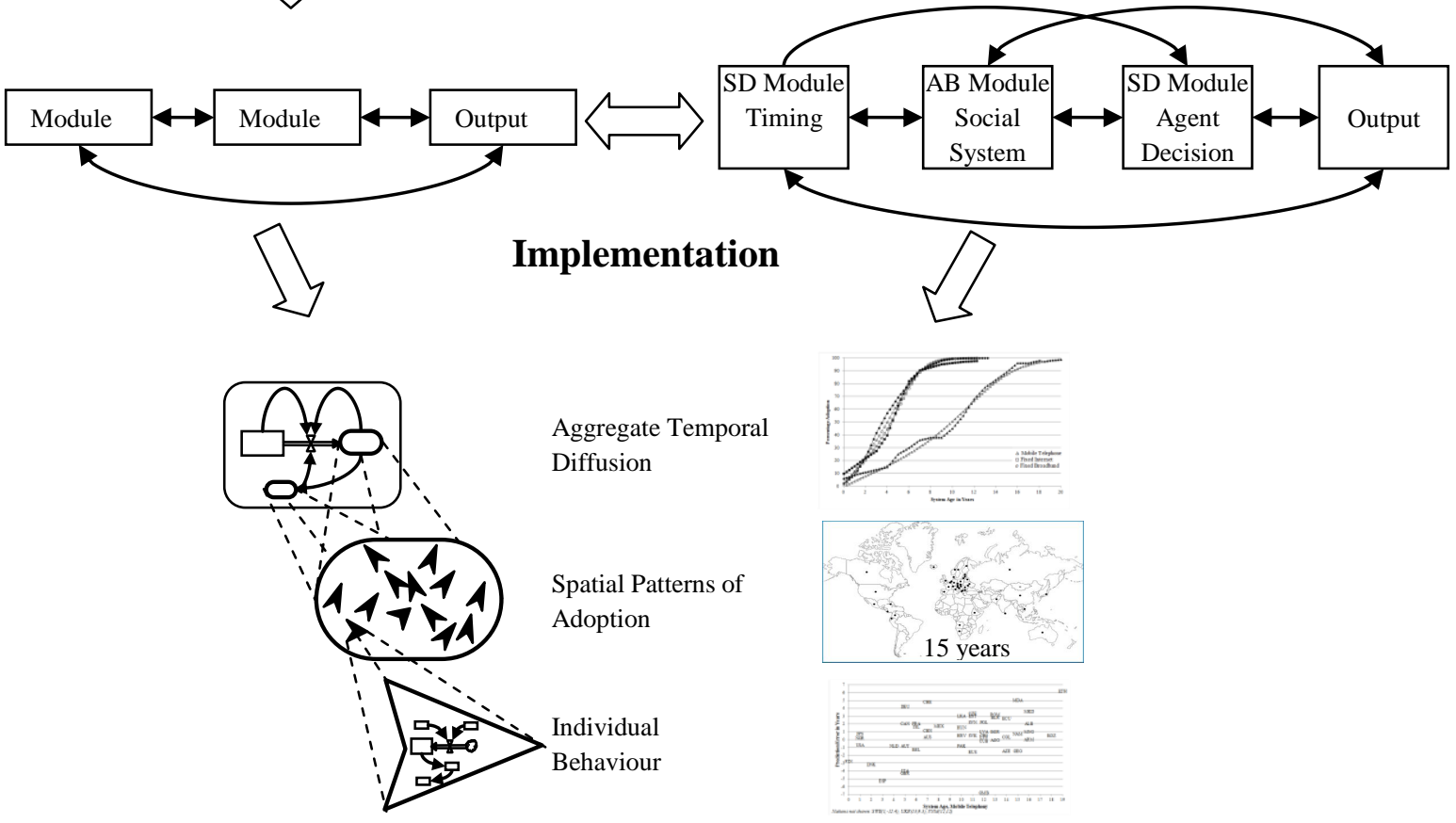

Figure 11 Summarising the general design process for hybrid modelling

\section{REFERENCES}

Bass F M (1969). A New Product Growth Model for Consumer Durables. Management Science 15(5): 215-227.

Chahal K, Eldabi T and Young T (2013). A conceptual framework for hybrid system dynamics and discrete event simulation for healthcare. Journal of Enterprise Information Management 26(1): 50-74.

Craig C S, Douglas S P and Grein A (1992). Patterns of Convergence and Divergence among Industrialised Nations: 1960 - 1988. Journal of International Business Studies; Fourth Quarter 1992; 23(4):773-787.

Dekimpe M G, Parker P M and Sarvary M (1998). Staged Estimation of International Diffusion Models An Application to Global Cellular Telephone Adoption. Technological Forecasting and Social Change 57(1-2): 105-132. 
Dekimpe M G, Parker P M and Sarvary M (2000). "Globalization”: Modeling Technology Adoption Timing Across Countries. Technological Forecasting and Social Change 63(1): 25-42.

Duggan J (2011). iSimPlus: A Multi-Method Simulation Tool for Modelling Complex Systems. Proceedings of the 29th International Conference of the System Dynamics Society. System Dynamics Society: Albany, NY.

Fishbein M and Ajzen I (1975). Belief, attitude, intention, and behavior: An introduction to theory and research. Reading, MA: Addison-Wesley.

Hamre L J (2008). Exploring the use of social capital to support technology adoption and implementation. DBA Thesis, University of Bath. http://opus.bath.ac.uk/, accessed 7 October 2011.

Kiesling E, Günther M, Stummer C and Wakolbinger L M (2012). Agent-based simulation of innovation diffusion: a review. Central European Journal of Operations Research 20(2): 183 230.

Parunak H V, Savit R, and Riolo R L (1998). Agent-Based Modelling Vs. Equation-Based Modelling: A Case Study and Users ' Guide. Springer-Verlag, Berlin, pp10-25.

Lane D C (1994). With a little help from our friends: how system dynamics and soft OR can learn from each other. System Dynamics Review 10(2-3): 101-134.

Lorenz $\mathrm{T}$ and Jost A (2006). Towards an orientation framework in multi-paradigm modeling. Proceedings of the 24th International Conference of the System Dynamics Society. System Dynamics Society: Albany, NY.

Rogers E M (2003). Diffusion of Innovations, New York: The Free Press. $5^{\text {th }}$ Edition.

Rahmandad H and Sterman J (2008). Heterogeneity and Network Structure in the Dynamics of Diffusion: Comparing Agent-Based and Differential Equation Models. Management Science Vol. 54(5): 998-1014.

Sailer L D (1978). Structural Equivalence: Meaning and Definition, Computation and Application*. Social Networks, 1(1): 73 - 90.

Sargent R G (2013). Verification and validation of simulation models. Journal of Simulation 7(1): 1224.

Shanthikumar JG and Sargent RG (1983). A unifying view of hybrid simulation / analytic models and modeling. Operations Research 31(6): 1030 - 1052.

Sterman J D (2000). Business Dynamics: Systems Thinking and Modelling for a Complex World. Irwin/McGraw-Hill: Chicago.

Swinerd C and McNaught K R (2012a). Design classes for hybrid simulations involving agent-based and system dynamics models, Simulat. Modell. Pract. Theory, Vol.20(1): 118-133; doi:10.1016/j.simpat.2011.09.002. 
Swinerd C and McNaught K R (2012b). Getting the most out of an international diffusion model through evolutionary programming, Proceedings of the 2012 Winter Simulation Conference. IEEE: Piscataway, NJ.

Taylor S and Todd P A (1995). Understanding Information Technology Usage: A Test of Competing Models. Information Systems Research 6(2): 144-176.

Venkatesh V, Smith R H, Morris M G, Davis G B, Davis F D and Walton S M (2003). User acceptance of information technology: toward a unified view. MIS Quarterly Vol. 27(3): 425478.

Viana J, Rossiter S, Channon A A, Brailsford S C and Lotery A (2012). A multi-paradigm, whole system view of health and social care for age-related macular degeneration. Proceedings of the 2012 Winter Simulation Conference. IEEE: Piscataway, NJ.

Wakeland W W, Gallaher E J, Macovsky L M and Aktipis C A (2004). A comparison of system dynamics and agent-based simulation applied to the study of cellular receptor dynamics. Proceedings of the 37th Annual Hawaii International Conference on System Sciences, Waikola, USA.

Wilensky U (1999). NetLogo. http://ccl.northwestern.edu/netlogo. Center for Connected Learning and Computer-Based Modeling. Northwestern University, Evanston, IL.

World Bank (2012). http://databank.worldbank.org/ddp/home.do, accessed 7 October 2011.

\section{AUTHOR BIOGRAPHIES}

CHRIS SWINERD is currently reading for a $\mathrm{PhD}$ in hybrid simulation modelling with Cranfield University. He received a BSc(Hons) Electronic Engineering from the University of Kent in 1991 and an MSc Signal Processing from the University of Surrey in 1994. He is a Principal Engineer with the Defence Science and Technology Laboratory, which is part of the Ministry of Defence.

KEN MCNAUGHT has a BSc (Hons.) in Physics and Astronomy from Glasgow University, an MSc in Operational Research from Strathclyde University and a $\mathrm{PhD}$ in Operational Research from Cranfield University. He leads the Operational and Decision Analysis Group at Cranfield University's Shrivenham campus. His current research interests include simulation, decision analysis and intelligent decision support systems. 